\title{
ORGANIZAÇÃO E ATENDIMENTO DE UMA UNIDADE BÁSICA DE SAÚDE: SIGNIFICADOS PARA USUÁRIOS/FAMILIARES E FUNCIONÁRIOS*
}

\author{
Fernanda Regina Vicente ${ }^{1}$, Grasiele Campregher ${ }^{2}$, Alacoque Lorenzini Erdmann ${ }^{2}$ Lucia Hisako Takase Gonçalves ${ }^{4}$
}

\begin{abstract}
RESUMO: Este estudo objetivou esclarecer os significados da organização e atendimento numa Unidade Básica de Saúde para seus funcionários e usuários/familiares. Ancorou-se nos fundamentos teórico-filosóficos e políticas de práticas no cuidado à saúde do SUS. Foi um estudo de abordagem qualitativa, tendo como sujeitos 10 funcionários e 20 usuários dos programas de saúde da UBS. As entrevistas e observações foram realizadas em junho e julho de 2007. Da análise dos dados, segundo Bardin, emergiram os temas referentes a Organização: Sobrecarga/demanda - ESF; Equipe multiprofissional; Funcionamento; Organização participativa; e Qualidade da organização. E, referentes ao Atendimento: Sobrecarga/ demanda; Referência e Contra-Referência/ Especialidades; Atenção Básica em Saúde; Fluxo ágil; Acolhimento; Comparação com outros serviços de saúde; e Qualidade do atendimento. Estes significados mostram a importância da organização e atendimento desta Unidade e contribuem para a melhoria da gestão dos serviços de saúde.
\end{abstract}

PALAVRAS-CHAVE: Serviços de Saúde; Enfermagem em Saúde Comunitária; Sistemas de Saúde.

\section{ORGANIZATION AND CARE DELIVERY AT A PRIMARY HEALTH CARE UNIT: MEANINGS FOR USERS/FAMILY MEMBERS AND STAFF}

\begin{abstract}
This study aimed to clarify the meanings of organization and care delivery at a Primary Health Care Unit for the staff and users / family members. It was based on the theoretical-philosophical and political practices of SUS (National Health System) health care. It was a study of qualitative approach, with 10 staff members and 20 users of health programs at the health care center. Interviews and observations were performed in June and July of 2007. From the data analysis, according to Bardin, the following themes concerning the Organization emerged: Overload/demand; multidisciplinary team; Operation; Participant Organization: quality of the organization. Referring to Care Delivery: Overload/demand; Reference and Counter-Reference/Specialties; Primary Health Care; Efficient Flow; Users' Welcoming; Comparison with other health services, and Quality of care delivery. These meanings show the importance of organization and care delivery in this unit and contribute to the improvement of health service management.
\end{abstract}

KEYWORDS: Health Services; Community Health Nursing; Health Systems.

\section{ORGANIZACIÓN Y CUIDADO DE UNA UNIDAD BÁSICA DE LA SALUD: SIGNIFICA- DOS PARA LOS USUARIOS/ FAMILIARES Y EMPLEADOS}

RESUMEN: Este estudio tuvo como objetivo aclarar el significado de organización y atención en una Unidad Básica de Salud (UBS) para empleados y usuarios/familia. Se apoyó en las bases teórico-filosóficas y políticas de prácticas en el cuidado de salud de SUS. Se trata de un estudio cualitativo, con 10 empleados y 20 usuarios de la UBS. Se realizó en junio y julio de 2007, despues de aprobado el proyecto por el Comité de Ética en la Investigación. En análisis de datos según Bardin, surgieron las cuestiones relativas a la organización: sobrecarga/demanda; equipo multidisciplinario; operación; organización de participación, cualidad de organización. En relación al cuidado: sobrecarga/demanda; referencia y contrareferencia/especialidades; atención básica de salud; de flujo ágil; acogimiento; comparación con otros servicios de salud, cualidad de atención. Estos significados mostran la importancia de organización y atención de esta Unidad y contribuyen para la mejoría de la gestión de los servicios de salud.

PALABRAS CLAVE: Servicios de salud; Enfermería en salud comunitária; Sistemas de salud.

\footnotetext{
*Artigo resultado da pesquisa de Conclusão de Curso de Graduação em Enfermagem. Universidade Federal de Santa Catarina-UFSC. ${ }^{1}$ Acadêmica do Curso de Graduação em Enfermagem da UFSC.

${ }^{2}$ Acadêmica do Curso de Graduação em Enfermagem da UFSC.

${ }^{3}$ Enfermeira. Doutora em Enfermagem. Docente do Departamento de Enfermagem e do Programa de Pós-Graduação em Enfermagem da UFSC.

${ }^{4}$ Enfermeira. Doutora em Enfermagem. Docente do Departamento de Enfermagem e do Programa de Pós-Graduação em Enfermagem da UFSC.
}

Autor correspondente:

Fernanda Regina Vicente

Rua Benjamin Constant, 322 - 89120-000 - Timbó-SC

Recebido: 06/02/08

Email: fernanda.frv@hotmail.com

Aprovado: 01/09/08 


\section{INTRODUÇÃO}

O Sistema Único de Saúde (SUS) foi criado pela Constituição Federal de 1988 e tem por finalidade tornar obrigatório e universal o atendimento público de saúde à população brasileira. O SUS teve seus princípios estabelecidos na Lei Orgânica de Saúde, em 1990, com base no artigo 198 da Constituição Federal, sendo esses princípios norteadores: universalidade, integralidade, eqüidade, participação da comunidade, descentralização, hierarquização e regionalização( ${ }^{(1)}$.

A regulamentação do SUS implica em condições para promoção, proteção e recuperação da saúde, a organização e o funcionamento dos serviços. Devido às características singulares da organização dos serviços de saúde e à diversidade da população, dos recursos econômicos e da capacidade gerencial dos municípios brasileiros, há a transferência do gerenciamento e organização dos serviços da União para os estados e dos estados para os municípios ${ }^{(2)}$.

A Atenção Básica é a forma de organizar o primeiro nível de atenção à saúde no SUS, estimulada pelo Ministério da Saúde. Insere-se no movimento mundial de valorização da atenção primária à saúde e na construção dos sistemas públicos de saúde ${ }^{(3)}$. Caracteriza-se por um conjunto de ações de saúde, no âmbito individual e coletivo, que abrangem a promoção e a proteção da saúde, a prevenção de agravos, o diagnóstico, o tratamento, a reabilitação e a manutenção da saúde. É desenvolvida por meio do exercício de práticas gerenciais e sanitárias democráticas e participativas, sob forma de trabalho em equipe, dirigidas a populações de territórios delimitados, pelas quais assume a responsabilidade sanitária, considerando a dinamicidade existente no território em que vivem essas populações. Utiliza tecnologias de elevada complexidade e baixa densidade, que devem resolver os problemas de saúde de maior freqüência e relevância em seu território. Orienta-se pelos princípios da universalidade, da acessibilidade e da coordenação do cuidado, do vínculo e continuidade, da integralidade, da responsabilização, da humanização, da eqüidade e da participação social ${ }^{(4)}$.

A Saúde da Família, outro programa do Ministério da Saúde, é entendida como uma estratégia de reorientação do modelo assistencial, operacionalizada mediante a implantação de equipes multiprofissionais em unidades básicas de saúde. Essas equipes são responsáveis pelo acompanhamento de um número definido de famílias, localizadas em uma área geográfica delimitada. As equipes atuam com ações de promoção da saúde, prevenção, recuperação, reabilitação dos usuários com doenças e agravos mais freqüentes e na manutenção da saúde desta comunidade ${ }^{(3)}$.

O Ministério da Saúde realiza vários programas com a missão de trazer a saúde para perto do cidadão e ensejar ao profissional a especialização necessária, a fim de que possa exercer seu trabalho com mais qualidade. Nesses programas devem ser observados os princípios básicos que regem a organização de um serviço de saúde, dentre eles o planejamento.

A estruturação de um serviço ocorre a partir do diagnóstico da realidade e de um planejamento adequado, considerando-se todas as variáveis possíveis nesse processo. A identificação e a análise do contexto da atenção à saúde e respectivos problemas podem subsidiar e qualificar as decisões e ações dos profissionais de saúde e gestores, de modo que haja participação, no intuito de alcançar metas com resolutividade, efetividade, considerando-se as vantagens e desvantagens para todos os envolvidos ${ }^{(5)}$.

Na gestão de uma Unidade Básica de SaúdeUBS é necessário se atentar para a organização da mesma e respectiva política de atendimento aos usuários. Nossas inquietações vão além da atividade técnica de fazer um adequado diagnóstico da realidade e respectivo planejamento com tomada de decisão participativa e resolutiva que atenda os princípios do SUS. São inquietudes voltadas para a expressão dos significados e, assim, permitam nos aproximar da compreensão das relações e interações vivenciadas pelas pessoas no cenário de uma UBS. Para tal, estabelecemos como objetivo deste estudo: esclarecer os significados da organização e atendimento ou cuidado prestado de uma Unidade Básica de Saúde, segundo seus funcionários e os usuários e familiares, bem como obter sugestões para melhoria da gestão da mesma.

\section{METODOLOGIA}

Esta pesquisa é do tipo exploratório-descritivo, de abordagem qualitativa com os usuários/familiares e funcionários da UBS do bairro Trindade, município de Florianópolis, Santa Catarina.

Os sujeitos desta pesquisa foram 10 funcionários e 20 usuários dos programas de saúde da UBS Trindade (Plano Nacional de Imunização, Atenção à Saúde da Criança, Programa de Atenção Integral à Saúde da Mulher, Programa de Doenças Sexualmente Transmissíveis, Programa Nacional de Controle da 
Tuberculose, Programa de Controle do Tabagismo, Programa de Atenção à Hipertensão Arterial e ao Diabetes Mellitus e Programa de Saúde do Idoso).

A escolha dos sujeitos de pesquisa ocorreu de forma intencional. Como critério para participar da pesquisa foram selecionados funcionários distribuídos em diferentes funções. Também foram entrevistados usuários dos diferentes programas de saúde implantados na Unidade.

A UBS Trindade subordina-se à Secretaria Municipal de Saúde de Florianópolis, e desenvolve programas vinculados à Estratégia Saúde da FamíliaESF. Essa Unidade tem como missão o desenvolvimento de ações de promoção da saúde para melhoria da qualidade de vida de sua comunidade, mediante o desenvolvimento de um modelo de atenção baseado na prevenção, diagnóstico, tratamento de doenças e recuperação, manutenção e promoção da saúde com participação da comunidade. Esse trabalho ocorre em conformidade com os princípios e diretrizes do SUS, com ações dirigidas aos indivíduos, à família e à comunidade.

Os bairros circunscritos à Unidade comportam uma população aproximada de 19.713 habitantes $^{(6)}$, e a mesma atende cerca de 2.567 famílias que estão distribuídas em 27 micro-áreas.

Os profissionais da UBS Trindade se distribuem em três equipes de ESF, que desempenham as seguintes atividades: consulta de enfermagem, médica, odontológica, psiquiátrica, fonoaudiológica e psicológica; procedimentos básicos de enfermagem; preventivo de câncer de colo uterino e mama; teste do pezinho; vacinação; desenvolvimento de grupos terapêuticos, grupos educativos, grupos de ajuda mútua; assistência farmacêutica; visitação domiciliar; visita a entidades comunitárias; vigilância epidemiológica, notificação compulsória e controle de tuberculose, entre outras.

A coleta de dados ocorreu no período de junho a julho de 2007, após a aprovação do projeto pelo Comitê de Ética em Pesquisa com Seres Humanos (Protocolo 064/07) e após a assinatura do Termo de Consentimento Livre e Esclarecido pelos sujeitos da pesquisa, seguindo as normas estabelecidas pela resolução nº 196/96 do Conselho Nacional de Saúde ${ }^{(7)}$.

Nestes preceitos éticos, a preocupação centrouse em garantir os princípios de beneficência, respeito à pessoa e justiça. Nesta garantia devem ser incluídas todas as pessoas que possam vir a ter alguma relação com a pesquisa, seja o sujeito da pesquisa, o pesquisador, o trabalhador das áreas onde a mesma se desenvolve e, em última análise, a sociedade como um todo ${ }^{(8)}$.

A coleta de dados foi realizada mediante entrevista e observação participante. As questões que nortearam as entrevistas com os funcionários foram: o que significa para você a organização (estrutura e funcionamento) e o atendimento (qualidade e quantidade) prestado nesta unidade em que trabalha? Que sugestões daria para melhorá-la? E, com os usuários e familiares, o que significa para você o atendimento ou cuidado recebido (qualidade e quantidade) e a organização (estrutura e funcionamento) desta unidade? Como gostaria que fosse?

Para a análise dos dados coletados, optamos por utilizar a análise de conteúdo de Bardin, do tipo análise temática. A análise de conteúdo é um conjunto de técnicas de análise das comunicações visando obter, por procedimentos sistemáticos e objetivos de descrição do conteúdo das mensagens, indicadores (quantitativos ou não) que permitam a inferência de conhecimentos relativos às condições de produção/ recepção (variáveis inferidas) destas mensagens ${ }^{(9: 31)}$. Esta abordagem permite explicitar e sistematizar o conteúdo das mensagens, possibilitando deduções lógicas e justificadas quanto à origem das mensagens do estudo. Para a utilização das falas dos informantes foram utilizadas as denominações "F" para funcionários e "U” para usuários/familiares.

\section{APRESENTANDO OS RESULTADOS}

Da análise dos dados emergiram os temas referentes à Organização da UBS: Sobrecarga/ demanda - ESF; Equipe multiprofissional da UBS; Funcionamento da UBS; Organização participativa; e Qualidade da organização. Referentes ao Atendimento aos usuários: Sobrecarga/demanda para atendimento; Referência e contra-referência ou especialidades; Atenção Básica em Saúde; Fluxo ágil no atendimento à demanda; Acolhimento dos usuários; Comparação com outros serviços de saúde; e Qualidade do atendimento.

Estes temas são descritos, a seguir, com a tentativa de mostrar como os dados apontam para uma consistência que possibilita discussões e reflexões, especialmente no tocante à política de promoção da saúde, sua filosofia em prol da autonomia dos sujeitos versus o caráter assistencialista que ainda prevalece na organização e no atendimento à saúde pelo SUS.

\section{Organização da UBS}




\section{Sobrecarga/demanda-ESF}

A sobrecarga de trabalho e a demanda dos usuários em uma ESF constituíram manifestações freqüentes entre funcionários, e também foram percebidas pelos usuários deste Programa:

Cada um desempenha a sua função e, muitas vezes, mais que a sua função. Então há uma sobrecarga. Com relação ao PSF, nós não damos conta de tudo. Nós somos 3 equipes para uma população que extrapola a quantidade para cada equipe, então a gente não consegue fazer um PSF como realmente deveria ser feito [...] (F.4).

Eu acho que no posto falta muita coisa para atender a grande demanda que temos (U.8).

Na ESF cada equipe se responsabiliza pelo acompanhamento de cerca de 3.000 a 4.500 pessoas ou de 1.000 famílias de uma determinada área e, estas passam a ter co-responsabilidade no cuidado à saúde ${ }^{(3)}$. Os bairros circunscritos à Unidade possuem uma população aproximada de 19.713 habitantes ${ }^{(6)}$. Baseando-se nessas informações, podemos confirmar que a população adscrita excede a capacidade de atendimento das equipes dessa Unidade, o que interfere negativamente na sua organização.

\section{Equipe multiprofissional da UBS}

Eu gostaria que a gente não tivesse estagiários ocupando o cargo de funcionários [...]. Eu penso que o estagiário é para dar suporte ou uma ajuda ao trabalho e não assumir o papel de um funcionário [...] mas, eu acho que isso é uma falha da organização (F.2).

Vários funcionários entrevistados queixaramse de que, com a falta de funcionários, os estagiários estão assumindo as responsabilidades destes, ocorrendo com isso um desfalque de profissionais capacitados na equipe. É oportuno lembrar que o estagiário realiza atividades de aprendizagem profissional e não pode assumir função de funcionário.

\section{Funcionamento da UBS}

Ficou mais viável para população. A população tem mais acesso agora. Tinha muita gente que nesse horário das oito da manhã às cinco da tarde não podia vir até aqui [...] (F.6).

Marcação de consulta, às vezes eu fico até me questionando, meu Deus! Mas todo mundo reclama que não tem consulta e é coisa que eu marco de uma semana para outra. O máximo que eu espero aqui é uma semana (U.17).

Na opinião dos funcionários a ampliação do horário de funcionamento da Unidade, que passou a ser das $7 \mathrm{~h}$ às $19 \mathrm{~h}$, melhorou o atendimento prestado à população podendo, assim, atender uma margem maior de usuários que eram prejudicados por trabalharem no horário de atendimento da Unidade.

As marcações de consultas com médico clínico geral não são levantadas como problemas pelos usuários da Unidade de Saúde, pois as consultas são agendadas dentro de uma semana. Podemos perceber o bom funcionamento das marcações de consultas médicas na Unidade de Saúde em estudo.

\section{Organização participativa}

Eu vejo que a coordenação coordena de uma forma horizontal, não é de cima para baixo. A busca da solução dos problemas vem de todos; todos buscam refletir, buscam participar, então é uma organização participativa. Todos participam da organização dessa unidade (F.10).

Através da fala deste funcionário percebemos que a coordenação da Unidade de Saúde tem papel fundamental na organização da mesma, possibilitando a abertura para demais funcionários participar da resolução dos problemas.

\section{Qualidade da organização}

Me surpreendeu, porque a gente sempre tem aquela visão que o serviço tem fila, é desorganizado e aqui é o contrário [...] aqui é muito organizado, desde a parte estética, que é uma clínica limpa, e o material. Quanto a respeito dos horários [...] nunca vim aqui e a consulta tava desmarcada ou o medicamento não tava (U.12).

Grande parte dos usuários entrevistados classificou a qualidade da organização da Unidade, considerando a estrutura e funcionamento como boa 
ou ótima. Com isso, é possível afirmar que a organização de qualidade reflete na satisfação dos usuários dos serviços de saúde.

\section{Atendimento aos usuários}

\section{Sobrecarga-demanda para atendimento}

É muita procura ainda prá quantidade de gente que tem disponível (F.1).

Considero que é um atendimento por recursos humanos bons. Não vejo, não costumo ver problemas maiores. A gente não tem demanda reprimida, assim, tem agendas abertas [...] (F.7).

Ocorre uma divergência de opiniões entre os funcionários a respeito da sobrecarga e demanda no atendimento. Essa divergência pode ser devido ao setor em que cada funcionário trabalha, possibilitando assim, o entendimento de que em determinado setor da Unidade ocorre sobrecarga de atendimento e em outro o atendimento não gera sobrecarga.

\section{Referência e contra-referência ou especialidades}

O grande nó está nas especialidades. Prá chegar no especialista demora e o paciente complica nesse meio tempo; e quando chega no hospital eles dizem que os postos de saúde não trabalharam direito. Não, nós fomos até onde pudemos e encaminhamos, e aí não dá prá dar continuidade (F.4).

Falta muita coisa, apesar da médica atender muito bem; ela receita, mas eu chego aqui, não tem a medicação, então a gente tem que comprar (U.15).

Para os funcionários o atendimento prestado em Atenção Básica de Saúde está adequado, porém quando o usuário necessita de um exame de alta complexidade ou de uma consulta com especialista o atendimento se torna demorado.

Uma das principais queixas dos usuários é a falta de medicação na farmácia da Unidade. Isso pode ser considerada uma falha na organização do fornecimento de medicação, que reflete no atendimento ao usuário.

\section{Atenção básica em saúde}

Em termos de SUS, o atendimento é ideal e realmente ele é atendido. Acredito que o usuário é atendido dentro das suas necessidades, porque a nossa Unidade presta serviço de atenção básica e acho que é o atendimento ideal na atenção básica (F.2).

Eu acho que, dentro do que preconiza o PSF, eu acredito que a gente está conseguindo desempenhar o papel principal de porta de entrada, prá todo o tipo de atendimento (F.3).

Como citado anteriormente, a Atenção Básica em Saúde realiza atividades de promoção e proteção da saúde, prevenção de agravos, diagnóstico, tratamento, reabilitação e manutenção da saúde, e todas essas ações são desenvolvidas na UBS Trindade, de acordo com os princípios do SUS.

\section{Fluxo ágil no atendimento da demanda}

A população chega aqui e sabe aonde ir e não fica perambulando prá lá e prá cá, logo é atendida; é muito difícil ser mandado embora [...] (F.9).

Tem alguma coisa a desejar. Principalmente a senha. Ali a senha, está com [...] confunde a farmácia com o atendimento ali na marcação de consultas. Certo? Então isso confunde. Podia ser diferenciado. É porque, às vezes, a pessoa nem sabe ler, não conhece números (U.8).

Segundo os funcionários, o atendimento da Unidade é ágil, pois o usuário não fica esperando muito tempo e é bem orientado quanto aos locais aos quais deve se dirigir. Quanto às opiniões dos usuários, tivemos algumas divergências, como reclamação da recepção, a forma como as senhas são distribuídas, a demora para atender às ligações telefônicas, entre outros.

\section{Acolhimento dos usuários}

A recepção ficou mais com o serviço dela que é atender telefone, avisar quando o paciente está aí prá consultar. Esse redimensionamento da organização refletiu muito na qualidade do atendimento. Tanto, que vejo a população mais satisfeita, do que há um ano atrás com o atendimento (após a implantação do acolhimento) (F.10).

Antes da implantação do acolhimento as pessoas se dirigiam à recepção para solicitar algum 
atendimento; agora, uma pessoa da equipe de saúde fica responsável por atender esse usuário que chega à Unidade e identificar a sua real necessidade.

O acolhimento, como postura e prática nas ações de atenção e gestão nas unidades de saúde, favorece a construção de uma relação de confiança e compromisso dos usuários com as equipes e os serviços, contribuindo para a promoção da cultura de solidariedade e para a legitimação do sistema público de saúde. Favorece, também, a possibilidade de avanços na aliança entre usuários, trabalhadores e gestores da saúde em defesa do SUS como uma política pública essencial da e para a população brasileira ${ }^{(10)}$.

\section{Comparação com outros serviços de saúde}

Os usuários se mostram, em sua maioria, satisfeitos com os serviços/atendimentos prestados na UBS Trindade. Podemos perceber isso nas comparações que fazem com relação a outros serviços de saúde:

Acho bem organizado. Em relação a [...] se você for comparar com outros postos de saúde eu acho bem organizado. A primeira vez que eu vim aqui até me surpreendeu, sinceramente (U.10).

Já tive planos de saúde e o atendimento não é aquilo que as pessoas pensam. Eu tinha que desembolsar todo mês e, além de tudo, ainda tinha que pagar. Você tem restrições demais nos planos de saúde que paga, onde não vale a pena (U.13).

\section{Qualidade do atendimento}

É a nossa razão de ser, o atendimento. E muitas vezes, a gente releva até situação de descontentamento com relação à administração, o salário, essas coisas todas e quantidade de pessoal $e$ até desvio de função em prol do bom atendimento. [...] em função desse atendimento mais qualificado até deixamos um pouco de lado a atividade do PSF na comunidade, prá atendimento na unidade (F.5).

Tô achando o atendimento perfeito, rápido, muito eficiente. Tenho a parabenizar o trabalho que tá sendo feito; em 5 minutos praticamente eu resolvi a situação, sem fila, sem problema nenhum. Se fosse de dar uma nota de 0 a 10, eu daria nota 10 (U.20).

A satisfação dos usuários com o atendimento prestado na Unidade supera os desagrados que, por ventura, são gerados. Os usuários mostram-se “felizes” com a atenção recebida pelos funcionários, e com a resolutividade de seus problemas. Os funcionários demonstram preocupação e interesse com o bem-estar desse usuário, justificando que o atendimento é a razão de existir do profissional de saúde.

\section{CONSIDERAÇÕES FINAIS}

Os significados dos temas destacados neste estudo mostram a importância da organização e atendimento nesta Unidade e representam situações significativas para os respondentes, os implicados no funcionamento da UBS. É imprescindível levar em consideração estes aspectos na seleção de estratégias de reorganização da gestão do serviço de saúde e do atendimento dos usuários da Unidade.

Portanto, conhecer os significados dados pelos implicados de uma UBS acerca de sua organização e assistência se constitui estratégia fundamental na avaliação e melhoria da gestão de uma UBS e, por extensão, na gestão dos serviços de saúde.

\section{REFERÊNCIAS}

1. Ministério da Saúde(BR). Lei $N^{\circ} 8.080$, de 19 de setembro de 1990. Constituição da República Federativa do Brasil [acesso em 2007 Jul 25]. Disponível em: www. portal.saude.gov.br

2. Martins SK. Diretrizes do atendimento domiciliar à saúde: contribuições da enfermeira [dissertação].Curitiba (PR): Universidade Federal do Paraná; 2006.

3. Ministério da Saúde (BR). Atenção básica [acesso em 2007 Mar 13]. Disponível em: www.saude.gov.br

4. Ministério da Saúde (BR). Política nacional de atenção básica. Série Pactos pela Saúde 2006, v.4. Brasília: Ministério da Saúde, 2006.

5. Kurcgant P. Gerenciamento em enfermagem. Rio de Janeiro: Guanabara Koogan; 2005.

6. IBGE. População unidade local de saúde Trindade de Florianópolis em 2002 [acesso em 2007 Mar 13]. Disponível em: www.pmf.sc.gov.br

7. Ministério da Saúde (BR). Manual operacional para comitês de ética em pesquisa. Série CNS - Cadernos técnicos. Brasília: Ministério da Saúde; 2002.

8. Goldim JR. Ética aplicada à pesquisa em saúde [acesso em 2007 Mar 20]. Disponível em: www.bioetica.ufrgs.br.

9. Bardin L. Analise de conteúdo. Lisboa: Edições 70; 1979.

10. Ministério da Saúde (BR). Acolhimento nas práticas de produção de saúde. Série B Textos básicos em saúde, $2^{\mathrm{a}}$ ed. Brasília; 2006. 